\title{
¿Debe recomendarse tratamiento citostático a un enfermo con carcinoma de páncreas metastásico?
}

El adenocarcinoma de páncreas (CP) representa la quinta causa de muerte por cáncer, de manera que tan sólo el 1-5\% de los pacientes permanecen vivos a los 5 años. Esto se debe a que en el momento del diagnóstico, el $80-90 \%$ de los enfermos padecen un tumor inoperable, bien sea por la extensión locorregional (30-40\%) o por presentar metástasis a distancia (50\%). Además, dado que el $80-90 \%$ de los pacientes con tumores localizados tratados quirúrgicamente van a recaer, puede afirmarse que bien en el momento del diagnóstico o bien tras la recaída, el 90-95\% de los enfermos con CP serán candidatos a recibir un tratamiento paliativo con quimioterapia (QT).

A lo largo de su evolución, el CP puede provocar la aparición de diferentes síntomas entre los que cabe destacar anorexia, pérdida de peso, astenia, dolor, naúseas y vómitos. Todo ello condiciona un deterioro de la calidad de vida, que se va acentuando a medida que progresa la enfermedad. Para completar este negro panorama, cabe recordar que la mediana de supervivencia de los enfermos con CP localmente avanzado es de 6-10 meses, y la de los pacientes con CP diseminado es de tan sólo 3-6 meses'.

Hasta hace algunos años, existía un pesimismo fundado sobre los posibles beneficios que podían derivarse de los tratamientos citostáticos en el CP. Este excepticismo tuvo su origen en el hecho habitual de que los buenos resultados que obtenía en algunas pequeñas series el tratamiento quimioterápico, luego no se confirmaban en los grandes estudios multicéntricos ${ }^{2}$. Por ello, y por la toxicidad asociada a estos tratamientos, diversos autores propusieron que no se debería tratar con quimioterapia (QT) a los pacientes con CP hasta que se demostrara de una forma convincente sus beneficios.

Así pues, es lícito preguntarse si actualmente se ha modificado esta situación. Sin embargo, aunque todavía siguen sin demostrarse estos beneficios de forma concluyente, diversos hechos han contribuido a modificar este panorama:

Servicio de Oncología Médica

Hospital La Paz

Madrid
1) La observación en algunos estudios aleatorizados de que la QT era eficaz para prolongar la supervivencia frente al tratamiento de soporte;

2) La comprobación de que la QT es capaz de inducir un beneficio clínico y aliviar determinados síntomas;

3) La demostración de que la QT, especialmente con los nuevos fármacos, puede conseguir reducciones objetivas del tumor. A continuación analizaremos con más detenimiento estos puntos.

\section{Impacto de la QT en la supervivencia}

Hasta la fecha se han publicado seis estudios aleatorizados en los que se comparó el tratamiento con QT frente a un grupo control sin ella ${ }^{5-10}$. Se observó que en tres de ellos, la QT conseguía prolongar significativamente la mediana de supervivencia ${ }^{3-5}$, mientras que en otros tres, no se detectaron diferencias $^{6-8}$ (Tabla I). Además, es justo reconocer que los resultados obtenidos en uno de esos estudios han sido muy cuestionados. En ese ensayo se empleó el denominado "régimen de Mallinson" compuesto por 5-Fluorouracilo (5FU), methotrexate, vincristina, ciclofosfamida y mitomicina $C$ y se logró un llamativo incremento de 8 meses en la mediana de supervivencia con respecto al grupo control ${ }^{3}$. Sin embargo, posteriormente se realizó otro ensayo clínico para confirmar estos resultados. En él se comparó ese mismo esquema con la administración de 5FU en monoterapia y con una combinación de 5FU-Adriamicina-Cisplatino. Se obtuvieron medianas de supevivencia de 4,5 meses para los dos primeros esquemas y de 3,5 meses para el último, sin que se encontraran diferencias estadísticas entre ellos 9 . Esto hizo que se cuestionara la validez de los resultados del primer estudio y que se considerara como tratamiento estándar el 5FU. En los otros dos estudios positivos, que son algo más recientes, el incremento de la supervivencia frente al grupo control fue más modesto y se situó en torno a los 4 meses. Además, en uno de ellos se observó que la QT no sólo lograba prolongar la supervivencia, sino que también conseguía mejorar la calidad de vida de los pacientes 5 .

En cualquier caso, conviene destacar que el número de enfermos incluidos en esos estudios fue muy reducido, por lo que su capacidad para detectar diferencias, en el caso de que existan, es también muy baja ${ }^{10}$. Por otra parte los fármacos utilizados en esos estudios actualmente no están con- 


\section{J. Feliú}

TABLA I

Estudios comparativos de quimioterapia frente a no tratamiento en el cáncer de páncreas avanzado

\begin{tabular}{|c|c|c|c|c|c|c|}
\hline Autor & Tratamiento & & $\begin{array}{c}\text { № de } \\
\text { enf }\end{array}$ & $\begin{array}{l}\text { Mediana } \\
\text { superv. }(\mathrm{m})\end{array}$ & $\begin{array}{c}P \\
6 \text { meses }\end{array}$ & $\begin{array}{l}\text { Supervivencia }(\%) \\
12 \text { meses }\end{array}$ \\
\hline $\begin{array}{l}\text { Mallinson } \\
\text { (1980) }\end{array}$ & $\begin{array}{l}\text { 5FU-MTX-VCR-CFM-MMC } \\
\text { Control }\end{array}$ & $\begin{array}{l}21 \\
19\end{array}$ & $\begin{array}{r}10.5 \\
2.2\end{array}$ & 0.000 & $\begin{array}{l}75 \\
12\end{array}$ & $\begin{array}{r}34 \\
6\end{array}$ \\
\hline $\begin{array}{l}\text { Andersen } \\
\text { (1981) }\end{array}$ & $\begin{array}{l}\text { 5FU-BCNU } \\
\text { Control }\end{array}$ & $\begin{array}{l}20 \\
20\end{array}$ & $\begin{array}{l}3.2 \\
3.4\end{array}$ & N.S. & $\begin{array}{l}20 \\
35\end{array}$ & $\begin{array}{l}10 \\
10\end{array}$ \\
\hline $\begin{array}{l}\text { Frey } \\
\text { (1981) }\end{array}$ & $\begin{array}{l}\text { 5FU-CCNU } \\
\text { Control }\end{array}$ & $\begin{array}{l}65 \\
87\end{array}$ & $\begin{array}{l}3.0 \\
3.9\end{array}$ & N.S. & $\begin{array}{l}18 \\
32\end{array}$ & $\begin{array}{r}8 \\
14\end{array}$ \\
\hline $\begin{array}{l}\text { Andrén } \\
\text { (1983) }\end{array}$ & $\begin{array}{l}\text { 5FU-CCNU-VCR } \\
\text { Control }\end{array}$ & $\begin{array}{l}25 \\
22\end{array}$ & $\begin{array}{l}6.5 \\
5.2\end{array}$ & N.S. & & \\
\hline $\begin{array}{l}\text { Palmer } \\
\text { (1994) }\end{array}$ & $\begin{array}{l}\text { 5FU-ADR-MMC } \\
\text { Control }\end{array}$ & $\begin{array}{l}23 \\
20\end{array}$ & $\begin{array}{l}8.2 \\
3.8\end{array}$ & 0.002 & $\begin{array}{l}70 \\
15\end{array}$ & $\begin{array}{r}25 \\
5\end{array}$ \\
\hline $\begin{array}{l}\text { Glimelius } \\
\text { (1996) }\end{array}$ & $\begin{array}{l}\text { 5FU-LV-VP16 } \\
\text { Control }\end{array}$ & $\begin{array}{l}39 \\
24\end{array}$ & $\begin{array}{r}6 \\
2.5\end{array}$ & 0.05 & $\begin{array}{l}50 \\
28\end{array}$ & $\begin{array}{l}23 \\
13\end{array}$ \\
\hline
\end{tabular}

5FU: 5-fluorouracilo; MTX: methotrexate; VCR: vincristina; CFM: ciclofosfamida; MMC: mitomicina C; ADR: adriamicina; LV: leucovorín

TABLA ॥

Beneficio clínico y tasa de respuestas inducidas por algunos esquemas de quimioterapia en el cáncer de páncreas

\begin{tabular}{|c|c|c|c|c|c|}
\hline Autor & Esquema & $(\%)$ & $\begin{array}{c}\text { Respuestas } \\
(\%)\end{array}$ & $\begin{array}{l}\text { Beneficio } \\
\text { clínico }\end{array}$ & $\begin{array}{c}\text { Mediana de la } \\
\text { supervivencia (meses) }\end{array}$ \\
\hline Burris & Gem & 63 & 5 & 24 & 5.6 \\
\hline (1997) & $5 \mathrm{FU}$ & 63 & 0 & 5 & 4.4 \\
\hline Colucci & CDDP-Gem & 53 & 26 & 53 & 4.7 \\
\hline (2002) & Gem & 54 & 9 & 49 & 7 \\
\hline $\begin{array}{l}\text { Hidalgo } \\
\text { (1999) }\end{array}$ & 5FU-Gem & 26 & 19 & 45 & 7.4 \\
\hline $\begin{array}{l}\text { Reni } \\
(2001)\end{array}$ & Gem-5FU-ADR-CDDP & 49 & 51 & 78 & 10 \\
\hline $\begin{array}{l}\text { Louvet } \\
\text { (2002) }\end{array}$ & Gem*-Oxal & 62 & 31 & 40 & 9.2 \\
\hline $\begin{array}{l}\text { Feliu } \\
\text { (2002) }\end{array}$ & UFT-Gem* & 43 & 33 & 64 & 11 \\
\hline $\begin{array}{l}\text { El-Rayes } \\
\text { (2003) }\end{array}$ & Gem-CDDP-5FU & 47 & 26 & - & 8.6 \\
\hline
\end{tabular}

*Gem en infusión de $120 \mathrm{mts}$.

Gem: Gemcitabina; 5FU: 5-Fluorouracilo; CDDP: Cisplatino, UFT : Uracilo-Tegafur; LV: leucovorín; Oxal: Oxaliplatino; ADR: Adriamicina.

siderados como los más activos, por lo que la magnitud de los beneficios que podría inducir un esquema más actual de QT sobre la supervivencia, probablemente fuera mayor que los que produjeron esos esquemas más antiguos.

\section{Quimioterapia y beneficio clínico}

Desde hace años se había observado que había enfermos en los que a pesar de que la QT no lograba reducir las di- mensiones del tumor, conseguía un alivio significativo de los síntomas. Para intentar cuantificar los posibles efectos del tratamiento sobre los síntomas y la calidad de vida de los pacientes con $\mathrm{CP}$, se desarrolló el concepto de beneficio clínico, que se basa en la medida de tres signos o síntomas muy habituales entre los pacientes con CP: dolor, estado funcional (performance status) y pérdida de peso. Para que se considerase que había habido beneficio clínico, era necesaria una mejoría en al menos uno de estos aspectos, mantenida durante 4 semanas y sin que empeorasen los otros pará- 
metros $^{11}$. A este respecto, resultan muy ilustrativos los resultados de un ensayo clínico realizado que comparó la administración de gemcitabina (GEM) frente a 5FU. En este estudio, a pesar de no detectarse diferencias en la tasa de respuestas ( $5 \%$ vs $0 \%$ ) (Tabla II), se observó que la GEM producía un beneficio clínico en el $24 \%$ de los enfermos frente al $5 \%$ del $5 F U(p<0.01)$. Además, hubo un discreto aumento de la supervivencia $(5,6$ vs 4,4 meses; $p<0.05)$ y en el tiempo libre de progresión (9 v.s. 4 semanas $p<0.001)^{11}$. A partir de este trabajo se redujo el tradicional excepticismo con el que se afrontaba la quimioterapia en el $C P$, aumentó el interés por el tratamiento de este tumor y se convirtió la GEM en el nuevo tratamiento estándar del CP. Posteriormente, otros autores han utilizado el beneficio clínico como objetivo terapéutico en sus ensayos clínicos, alcanzando mejorías en el 20-50\% de los enfermos ${ }^{11-17}$. Cabe insistir en que a menudo estos beneficios se obtienen sin que se produzca una reducción objetiva de las dimensiones del tumor, sino que mínimas modificaciones, no detectables por TAC, pueden ser suficientes para que disminuya la presión que ejerce el tumor sobre el plexo celiaco y, por consiguiente, para reducir la intensidad de los síntomas. Por otra parte, la consideración del beneficio clínico como objetivo terapéutico tiene el inconveniente de basarse en la valoración de datos subjetivos, con lo poco precisa que resulta su determinación a pesar del empleo de escalas estandarizadas. En cualquier caso y aún reconociendo estas limitaciones, lo cierto es que cada vez hay más estudios en el CP que consideran al beneficio clínico como principal objetivo terapéutico, probablemente por la dificultad para demostrar una mejora en la supervivencia o en las respuestas radiológicas.

\section{Actividad de la quimioterapia sobre el tumor}

Tradicionalmente, uno de los principales objetivos de los tratamientos en oncología es conseguir una reducción en el tamaño del tumor. Sin embargo, en el CP, hasta la incorporación del TAC, era prácticamente imposible valorar el tamaño del tumor, por lo que las tasas de respuesta que se comunicaban eran poco fiables. Incluso ahora sigue siendo complicado valorar las respuestas radiológicas que experimentan los tumores de páncreas aunque se emplee el TAC, los ultrasonidos o la resonancia magnética. Conviene recordar que el CP, a menudo está compuesto, tanto por tejido tumoral como por células inflamatorias y estromales, de manera que comúnmente el componente inflamatorio supera al tumoral. Por ello, la QT podría destruir células tumorales sin que se modifiquen las imágenes radiológicas, ya que es difícil obtener regresiones rápidas de los tejidos fibróticos, mientras que por el contrario, tratamientos antiinflamatorios (corticoides...) podrían lograr respuestas radiológicas al disminuir el componente inflamatorio del tumor, pero sin que ésto signifique que se ha conseguido reducir el tejido tumo$\mathrm{ral}^{2}$. Por todo ello, parece más apropiado analizar la respuesta considerando las metástasis y no el tumor primitivo.

En cualquier caso y teniendo en cuenta estas consideraciones, cabe destacar que con los esquemas de QT más modernos se alcanzan tasas de respuesta que oscilan entre el 10 y el $51 \% 11-17$.

Con respecto a los posibles esquemas a utilizar, cabe señalar que todavía siguen vigentes los resultados del ensayo en fase III anteriormente citado, en el que se comparó su administración en monoterapia con la de 5FU, por lo que todavía se puede considerar a la GEM como el tratamiento de referencia ${ }^{18}$. Sin embargo, aunque la GEM produce un claro beneficio clínico y una mínima prolongación de la supervivencia, apenas obtiene reducciones objetivas del tumor y la mediana de supervivencia sigue siendo muy corta $(<6$ meses). Más recientemente, se ha sugerido que su eficacia terapéutica puede mejorarse cuando se administra como una infusión a un ritmo fijo de $10 \mathrm{mg} / \mathrm{m}^{2} / \mathrm{mt}$. Esto permite aumentar la concentración de los metabolitos activos de la GEM en la célula tumoral, y así incrementar su actividad. Se realizó un ensayo en fase ll aleatorizado en el que se comparó GEM en monoterapia a dosis de $2.200 \mathrm{mg} / \mathrm{m}^{2}$ en 30 minutos frente a GEM $1500 \mathrm{mg} / \mathrm{m}^{2}$ administrada en 150 minutos. Se observaron tasas de respuestas del $3 \%$ y del $17 \%$ respectivamente. Por su parte, la mediana de supervivencia fue de 5 v.s. 8 meses, con un $9 \%$ v.s. $28 \%$ de supervivientes al año y un $2 \%$ y $18 \%$, respectivamente, a los dos años ${ }^{19}$.

Igualmente, para intentar mejorar los resultados que se logran con la GEM en monoterapia, se ha estudiado su combinación con otros fármacos. Las combinaciones de GEM con fluoropirimidinas orales, como UFT, capecitabina o 5FU se toleran bien, pero los estudios randomizados no han conseguido demostrar que aumente la supervivencia sobre la GEM $^{20}$. Igualmente, las combinaciones GEM-irinotecán ${ }^{21}$ y GEM-cisplatino ${ }^{12}$, consiguieron un incremento en la tasa de respuestas pero no en la supervivencia. Más prometedores son los resultados comunicados con el esquema GEMOX (Gem-Oxal). En un estudio comparativo frente a Gem se observó un incremento en la tasa de respuestas ( $26 \%$ vs $16 \%$ ), beneficio clínico (39\% vs $28 \%$ ) y tiempo libre de progresión ( $6 \mathrm{~m}$ vs $4 \mathrm{~m}$ ). Todavía se está pendiente de resultados de supervivencia ${ }^{22}$. Así pues, aunque todavía puede considerarse a la GEM como el tratamiento estándar, la adición de otros fármacos permite aumentar la tasa de respuesta y el beneficio clínico.

Puede concluirse afirmando que, a la vista de los estudios anteriormente expuestos, en la actualidad hay evidencias suficientes para recomendar a todo enfermo con CP avanzado y buen estado general (ECOG PS 0-2), el tratamiento con quimioterapia.

\section{Bibliografía}

1. Haller DG. New prspectives in the management of pancreas cancer. Semin Oncol 2003; 30 (suppl 11):3-10.

2. Popescu RA, Cunningham D. Chemotherapy for advanced pancreatic cancer- some light at the end of the tunnel? Ann Oncol 1997; 8:415-6.

3. Mallinson CN, Rake MO, Cocking JB, et al. Chemotherapy in pancreatic cancer: results of a controlled, prospective, randomised, multicentre trial. BMJ 1980; 281:1589-91.

4. Palmer KR, Kerr M, Knowles $G$, et al. Chemotherapy prolongs survival in inoperable pancreatic crcinoma. Br J Surg 1994; $81: 882-5$.

5. Grimelius B, Hoffman K, Sjoden PO, et al. Chemotherapy improves survival and quality of life in advanced pancreatic and biliary cancer. Ann Oncol 1996; 7:593-600.

6. Andersen JR, Friis-Moller A, Hancke S. A controlled trial of 


\section{J. Feliú}

combination chemotherapy with 5 -FU and BCNU in pancreatic cancer. Scand J Gastroenterol 1981; 16:973-5.

7. Frey C, Twomey P, Keehn R. Randomised study of 5-FU and CCNU in pancreatic cancer: Report of the Veterans Administration Surgical Cancer Chemotherapy Study Group. Cancer $1981 ; 47: 27-31$

8. Anden-Sandberg A, Holmberg JT, Ihse I. Treatment of unresectable pancreatic carcinoma with 5-fluorouracil, vincristine, and CCNU. Scand J Gastroenterol 1983; 18:609-12.

9. Cullinan S, Moertel CG, Wieand et al. A phase III trial on the therapy of advanced pancreatic carcinoma. Cancer 1990; 65:2207-12.

10. Permert J, Hafström L, Nygren P, Grimelius B. A syłematic overview of chemotherapy effects in pancreatic cancer. Acta Oncológica 2001; 40:361-70.

11. Burris III HA, Moore MJ, Andersen J, et al. improvements in survival and clinical benefit with Gemcitabine as first-line therapy for patients with advanced pancreas cancer: a randomized trial. J Clin Oncol 1997; 15:2403-13.

12. Colucci G, Giuliani F, Gebbia V, et al. Gemcitabine alone or with cisplatin for the treatment of patients with locally advanced and/or metastatic pancreatic carcinoma. A prospective, randomized phase III study of the Gruppo Oncologico dellítalia Meridionale. Cancer 2002; 94:902-10.

13. Hidalgo $M$, Castellano D, Paz-Ares $L$, et al. Phase I-II study of gemcitabine and fluorouracil as a continuous infusion in patients with pancreatic cancer. J Clin Oncol 1999; 17:585-92.

14. Reni M, Passoni $P$, Panucci MG, et al. Definitive results of a phase II trial of cisplatin, epirubicin, continuous-infusion fluorouracil, and gemcitabine in stage IV pancreatic adenocarcinoma. J Clin Oncol 2001; 19:2679-86.
15. Louvet $C$, André T, Lledo $G$ et al. Gemcitabine-Oxaliplatin (GEMOX) combination in advanced pancreatic carcinoma (APC): a Gercor multicenter phase II study. J Clin Oncol 2002; 20:1512-8.

16. Feliu J, Mel R, Borrega $P$, et al. Phase II study of a fixed doserate infusion of gemcitabine associated to UFT in advanced carcinoma of the pancreas. Ann Oncol 2002; 13:1756-62.

17. El-Rayes BF, Zalupski MM, Shields AF, et al. Phase II study of gemcitabine, cisplatin and infusional fluorouracil in advanced pancreatic cancer. J Clin Oncol 2003; 21:2920-5.

18. Kulke MH. Advanced pancreatic cancer: is there a role for combination therapy? Expert Rev. Anticancer Ther 2003; 3:729-39.

19. Tempero M, Plunkett W, Ruiz van Haperen V, et al. Randomized phase II comparison of dose-intense gemcitabine: thirty-minute infusion and fixed dose rate infusion in patients with pancreatic adenocarcinoma. J Clin Oncol 2003; 21:3402-8.

20. Berlin J, Catalano P, Thomas J, et al. A phase III study of gemcitabine in combination with 5 -FU vs. Gemcitabine alone in patients with advanced pancreatic carcinoma: Eastern Cooperative Oncology Group Trial E2297. J Clin Oncol 2002; 20:3270-5.

21. Rocha-Lima $C$, Rotche $R$, Jeffery $M$, et al. A randomized phase III study comparing efficacy and safety of gemcitabine and irinotecan to gemcitabine alone in patients with locally advanced or metastatic pancreatic cancer who have not received prior systemic therapy. Proc Am Soc Clin Oncol 2003; 22: 251 labst 1005).

22. Louvet $\mathrm{CH}$, Labianca $R$, Hammel $P$, et al. Gemcitabine versus GEMOX (Gemcitabine+Oxaliplatin) in non resecyable pancreatic adenocarcinoma: a GERCOR / GISCAD Intergroup phase III. Proc ECCO 12 2003; 1:S18 (abst 47). 